\title{
Zooplankton communities and their relationship with water quality in eight reservoirs from the midwestern and southeastern regions of Brazil
}

\author{
P. H. S. Picapedra ${ }^{a *}$ (D), C. Fernandes ${ }^{b}$ (D), G. Baumgartner B,b (D) and P. V. Sanches $^{a, b, c}$ \\ anniversidade Estadual do Oeste do Paraná - UNIOESTE, Programa de Pós-graduação em Recursos Pesqueiros e \\ Engenharia de Pesca, Toledo, PR, Brasil \\ ${ }^{\mathrm{b}}$ Instituto Neotropical de Pesquisas Ambientais - INEO, Grupo de Pesquisas em Recursos Pesqueiros e Limnologia - \\ GERPEL, Toledo, PR, Brasil \\ 'Universidade Estadual do Oeste do Paraná - UNIOESTE, Programa de Pós-graduação em Ciências Ambientais, \\ Toledo, PR, Brasil \\ *e-mail: pablo_picapedra@hotmail.com
}

Received: October 16, 2019 - Accepted: March 18, 2020 - Distributed: August 31, 2021

(With 4 figures)

\begin{abstract}
Zooplankton are widely recognised as being regulated primarily by predators and food availability. In reservoirs, the quantity and quality of food resources are generally affected by the characteristics of the water, which in turn are controlled by the flow pulse generated by operation of the dams. In this study, we investigated the relationship between zooplankton, water quality and food availability (phytoplankton) in eight hydroelectric reservoirs located in Brazil. Samples were collected during the rainy and dry periods between 2008 and 2009. In general, the reservoirs exhibited mesotrophic conditions and Cyanobacteria were the predominant phytoplankton. The results showed that the rotifers Kellicottia bostoniensis, Hexarthra mira, Keratella spp., and Polyarthra vulgaris were present, indicating nutrient-rich environments. In addition, the copepod Thermocyclops decipiens occurred in eutrophic environments. In contrast, the cladoceran Daphnia gessneri and copepod Notodiaptomus henseni were considered indicators of more desirable water quality, owing to their relationship with waters with lower levels of nutrients and suspended solids. The results support the use of these organisms as a useful tool for understanding changes in water quality and in the ecosystem processes involved.
\end{abstract}

Keywords: Cyanobacteria, nutrients content, plankton communities, trophic state, water quality indicator.

\section{Comunidades de zooplâncton e sua relação com a qualidade da água em oito reservatórios das regiões Centro-Oeste e Sudeste do Brasil}

\section{Resumo}

O zooplâncton é amplamente reconhecido como sendo regulado principalmente por predadores e pela disponibilidade de alimento. Em reservatórios, a quantidade e a qualidade de recursos alimentares são afetadas pelas características da água que, por sua vez, são controladas pelo pulso de fluxo gerado pela operação das barragens. Neste estudo, investigamos a relação entre o zooplâncton, qualidade d'água e a disponibilidade de alimento (fitoplâncton) em oito reservatórios hidrelétricos localizados no Brasil. Amostras foram coletadas durante os períodos chuvoso e seco, entre os anos de 2008 e 2009. Em geral, os reservatórios exibiram condições mesotróficas e Cyanobacteria foi o fitoplâncton predominante. Os resultados mostraram que os rotíferos Kellicottia bostoniensis, Hexarthra mira, Keratella spp. e Polyarthra vulgaris foram indicadores de ambientes ricos em nutrientes. Além disso, o copépode Thermocyclops decipiens ocorreu em ambientes eutróficos. Por outro lado, o cladócero Daphnia gessneri e o copépode Notodiaptomus henseni foram considerados indicadores de melhor qualidade da água, devido a sua relação com águas com baixos níveis de nutrientes e sólidos em suspensão. Os resultados suportam o uso desses organismos como uma ferramenta útil para o entendimento das mudanças na qualidade d'água e nos processos ecossistêmicos envolvidos.

Palavras-chave: Cyanobacteria, teor de nutrientes, comunidades planctônicas, estado trófico, indicador de qualidade da água. 


\section{Introduction}

Reservoirs are now inseparable components of the Brazilian landscape and are present in all the main hydrographic basins as a result of the choice made by the country to generate hydroelectricity (Takahashi et al., 2009; Simões et al., 2015). There has been an increased proliferation of these engineering works, which are important for the national energy matrix (Agostinho et al., 2007). In addition, they are considered of extreme importance for regional socio-economic development due to their multiple uses, including water supply, irrigation, aquaculture, and recreation, which increases the importance of studying these systems (Soares et al., 2008). Dams significantly alters riverside ecosystems because the creating of a reservoir blocks the free flow of the river and creates a semi-lentic or lentic habitat. Thus, important factors such as the quantity and quality of water, habitats, and nutrient transport can change dramatically (Baumgartner et al., 2017; Loken et al., 2018). In addition, artificial variations in the water level can directly and indirectly affect zooplankton communities.

Due to their short life cycle and the sensitivity of some species to environmental changes, zooplankton organisms are considered bioindicators in aquatic ecosystems (De-Carli et al., 2018). Zooplankton play an important role in energy transfer in these ecosystems, as well as in the maintenance and orientation of trophic nets (Eskinazi-Sant'Anna et al., 2013). In addition, due to its position in the food chain with close links with primary producers, changes in the phytoplankton community are quickly reflected in the structure of zooplankton (e.g., in their abundance, body size, and productivity) (Bonecker and Aoyagui, 2005; Serafim-Júnior et al., 2010; Brito et al., 2016).

A recurring problem in Brazilian reservoirs is the eutrophication process, attributed mainly to industrialisation, urbanisation, and the extensive use of the reservoir basins for livestock and agriculture, all of which compromises water quality (Brito et al., 2011). In the long term, eutrophication will cause drastic changes to community structures and aquatic food chains, leading to a loss of biodiversity and reducing the utility of reservoirs as well as their fish stocks (Agostinho et al., 2007). One of the effects associated with eutrophication is the dominance of phytoplankton by Cyanobacteria (Soares et al., 2009; Silva et al., 2014). During flowering events, Cyanobacteria can form large colonies or clusters that may interfere directly with the filtration apparatus of zooplankton (De-Mott et al., 2001). In this way, powerful mechanisms, such as the predominance of Cyanobacteria, can affect the structure of zooplankton communities, making zooplankton key to understanding changes in aquatic ecosystems, especially in understanding how these changes are propagated along the food chain (Silva et al., 2014; Perbiche-Neves et al., 2016).

In this context, we aim to identify the main factors that influence the quantitative structure of the zooplankton community in eight Brazilian hydroelectric reservoirs. Therefore, we aim to answer the following questions: (i) what are the seasonal dynamics of zooplankton in the different reservoirs? (ii) Do the quality and availability of food (phytoplankton) and limnological conditions influence the characterisation of zooplankton in the different reservoirs?

\section{Material and Methods}

\subsection{Study area}

This study was conducted at eight hydroelectric reservoirs located in the midwestern and southeastern regions of Brazil: midwestern region, Corumbá (COR) and Itumbiara (ITU); southeastern region, Funil (FUN), Furnas (FUR), Luiz Carlos Barreto de Carvalho (LBC), Marimbondo (MAR), Mascarenhas de Moraes (MSM), and Porto Colômbia (PCO) (Table 1; Figure 1). The COR $\left(17^{\circ} 59^{\prime} \mathrm{S} ; 48^{\circ} 31^{\prime} \mathrm{W}\right)$ and ITU $\left(18^{\circ} 24^{\prime} \mathrm{S} ; 4^{\circ} 05^{\prime} \mathrm{W}\right)$ reservoirs lie in the Paraná-Paraguay basin, and began operations in 1997 and 1980 , respectively. The MAR $\left(20^{\circ} 18^{\prime} \mathrm{S} ; 49^{\circ} 11^{\prime} \mathrm{W}\right)$, PCO (20 $\left.07^{\prime} \mathrm{S} ; 48^{\circ} 34^{\prime} \mathrm{W}\right)$, LBC $\left(20^{\circ} 09^{\prime} \mathrm{S} ; 47^{\circ} 16^{\prime} \mathrm{W}\right)$, MSM (20 $\left.16^{\prime} \mathrm{S} ; 47^{\circ} 03^{\prime} \mathrm{W}\right)$, and FUR (20 $\left.39^{\prime} \mathrm{S} ; 46^{\circ} 18^{\prime} \mathrm{W}\right)$ reservoirs are located along the Grande River, also lie in the Paraná-Paraguay basin, and began operations in 1975, $1973,1969,1957$, and 1963, respectively. The FUN reservoir $\left(22^{\circ} 35^{\prime} \mathrm{S} ; 44^{\circ} 35^{\prime} \mathrm{W}\right)$ is located on a river that drains waste from a densely populated and industrialised area in the Paraíba do Sul River basin, and began operation in 1969 (Soares et al., 2008).

Table 1. Characteristics of the eight Brazilian hydroelectric reservoirs.

\begin{tabular}{lccccccc}
\hline \multicolumn{1}{c}{ Reservoirs } & Abbreviation & River & $\begin{array}{c}\text { Area } \\
\left.\mathbf{( k m}^{\mathbf{2}}\right)\end{array}$ & $\begin{array}{c}\text { Volume } \\
\left(\mathbf{( k m}^{\mathbf{3}}\right)\end{array}$ & $\begin{array}{c}\mathbf{Z}_{\text {max }} \\
(\mathbf{m})\end{array}$ & $\begin{array}{c}\text { Discharge } \\
\left(\mathbf{m}^{\mathbf{3}} \mathbf{s}^{-\mathbf{}} \mathbf{)}\right.\end{array}$ & $\begin{array}{c}\text { WRT } \\
(\mathbf{d a y s})\end{array}$ \\
\hline Corumbá & COR & Corumbá & 55 & 1.2 & 60 & 475 & 51.1 \\
Funil & FUN & Paraíba do Sul & 27 & 0.5 & 45 & 202 & 32.9 \\
Furnas & FUR & Grande & 1,322 & 20.2 & 127 & 910 & 405.4 \\
Itumbiara & ITU & Paranaíba & 684 & 15.1 & 93 & 1,487 & 127.8 \\
Luiz Carlos Barreto de Carvalho & LBC & Grande & 45 & 1.3 & 57 & 951 & 1.8 \\
Marimbondo & MAR & Grande & 438 & 6.2 & 90 & 1,502 & 37.2 \\
Mascarenhas de Moraes & MSM & Grande & 248 & 3.7 & 55 & 967 & 51.1 \\
Porto Colômbia & PCO & Grande & 143 & 1.5 & 35 & 777 & 13.9 \\
\hline
\end{tabular}

Abbreviations: $Z_{\max }=$ maximum depth, $\mathrm{WRT}=$ water residence time 


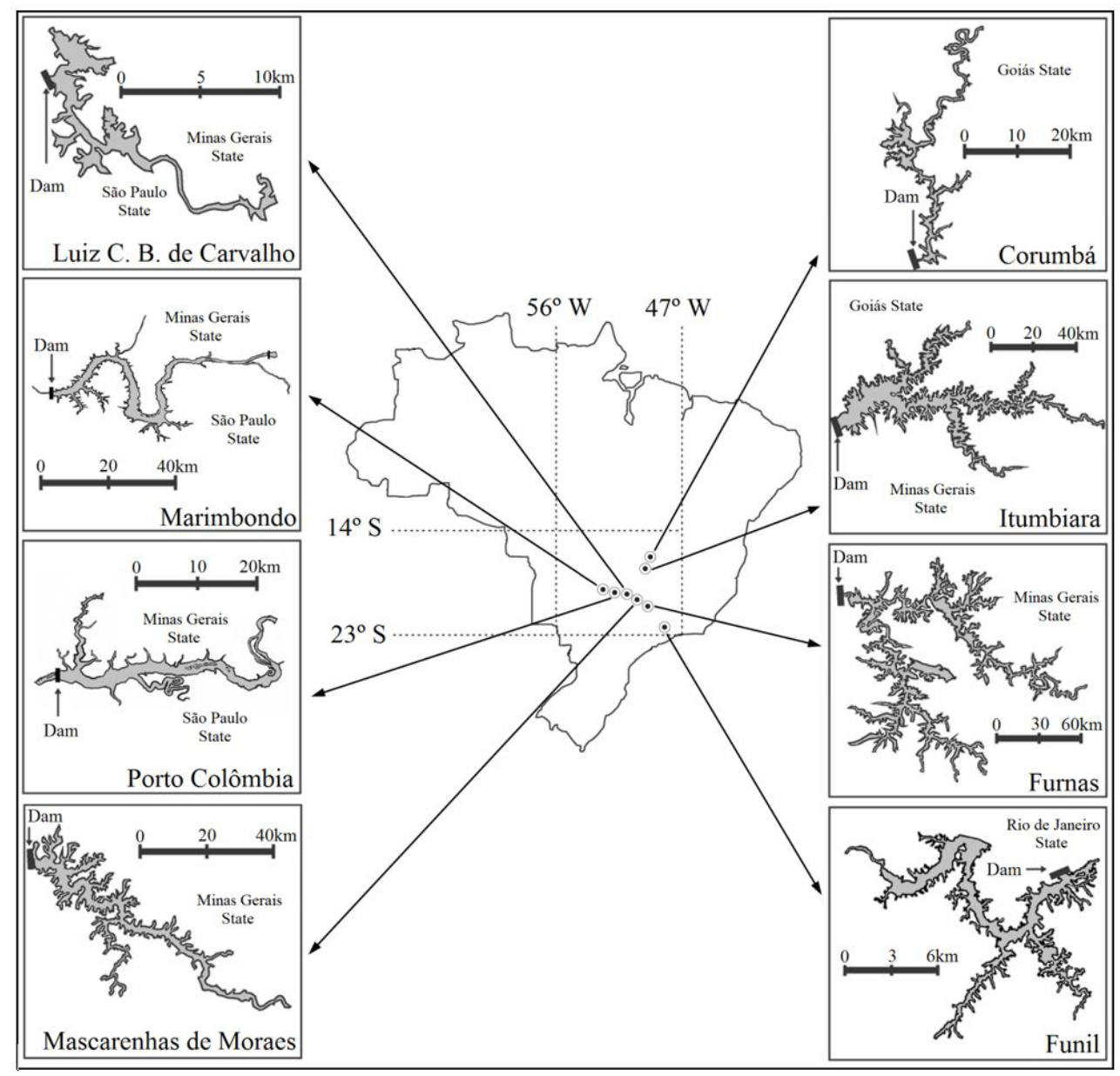

Figure 1. Map of Brazil showing the locations of the eight hydroelectric reservoirs.

\subsection{Sampling and data collection}

All reservoirs were sampled along the fluvial, transitional, and lacustrine zones for two months for each hydrological period (rainy and dry) between 2008 and 2009. The rainy period (December and February) was the hottest and covered the beginning and end of the rainy season, and the dry period (June and August) corresponded with mild temperatures. The study was repeated twice, spaced two months apart, to reduce the disturbance effects of sampling. Water samples were collected from the subsurface for the analysis of limnological variables: total phosphorus (TP, $\mathrm{mg} \mathrm{L}^{-1}$ ), total nitrogen ( $\mathrm{TN}, \mathrm{mg} \mathrm{L}^{-1}$ ), total suspended solids (TSS, $\mathrm{mg} \mathrm{L}^{-1}$ ), and chlorophyll $a\left(\mathrm{Chl}-a, \mu \mathrm{g} \mathrm{L}{ }^{-1}\right)$ concentrations. Water temperature (Temp, $\left.{ }^{\circ} \mathrm{C}\right)$, dissolved oxygen $\left(\mathrm{O}_{2}, \mathrm{mg} \mathrm{L}^{-1}\right)$, electrical conductivity (Cond, $\mu \mathrm{S} \mathrm{cm}^{-1}$ ), and $\mathrm{pH}$ data were obtained using a multiparameter probe. For phytoplankton, $250 \mathrm{~mL}$ of water was sampled from the subsurface with a polypropylene flask, and the material was fixed with Lugol's solution. Quantitative zooplankton samples were collected on the subsurface using a motorised pump to filter $200 \mathrm{~L}$ of water per sample, with a conical-cylindrical net $(63 \mu \mathrm{m}$ mesh). The collected material was packed in polyethylene flasks $(500 \mathrm{~mL})$, labelled, and fixed in $4 \%$ formaldehyde buffered with sodium borate $\left(\mathrm{Na}_{3} \mathrm{BO}_{3}\right)$.

\subsection{Sample analysis}

The preserved water samples were analysed for their Chl- $a$, TP, TN, and TSS concentrations according to Apha (2005). Phytoplankton (Cyanobacteria + algae, cells $\mathrm{mL}^{-1}$ ) were quantified in random fields using the settling technique (Utermöhl, 1958). The units (cells, colonies, and filaments) were quantified for at least 100 specimens of the most frequent species (Lund et al., 1958) under an inverted microscope at $400 \times$ magnification. The zooplankton (individuals $\mathrm{m}^{-3}$ ) were quantified in a Sedgewick-Rafter counting chamber. Aliquots for counting were removed from the samples with a standardised volume $(50 \mathrm{~mL})$ using a Hensen-Stempel pipette $(2.5 \mathrm{~mL})$. At least 50 individual rotifers, cladocerans, and juvenile (nauplii and copepodites) and adult copepods were counted (adapted from Bottrell et al., 1976) under a microscope, with a magnification range of $10 \times$ to $100 \times$. The species were identified according to Koste (1978), Reid (1985), Matsumura-Tundisi (1986), and Elmoor-Loureiro (1997). 


\subsection{Data analysis}

The Trophic State Index (TSI) was calculated according to Equation 1, 2 and 3 (modified by Lamparelli, 2004), considering the Chl- $a$ and TP values (Table 2):

$\operatorname{TSI}(C h l-a)=10 \times\{6-[(0.92-0.34) \times(\ln C h l-a)]\} / \ln 2$

$\operatorname{TSI}(T P)=10 \times\{6-[(1.77-0.42) \times(\ln T P)]\} / \ln 2$

$T S I=[T S I(C h l-a)+T S I(T P)] / 2$

A one-way analysis of variance (ANOVA) was used, with a significance level of $p<0.05$, to investigate the differences in limnological variables, phytoplankton, and zooplankton abundance between periods (rainy and dry). For this analysis, data from the three environments of each reservoir were grouped. Normality and homoscedasticity (homogeneity of variance) were initially verified using the Shapiro-Wilk and Levene tests, respectively. The relationship between the limnological variables, phytoplankton, and zooplankton taxa was explored using canonical correspondence analysis (CCA) for each hydrological period. The statistical significance of the eigenvalues and the species-environment correlations for the axes generated by the CCA were tested by the Monte Carlo method based on 999 permutations (Legendre et al., 2011), with a significance level of $p<0.05$.

All data (except $\mathrm{pH})$ were $\log (x+1)$ transformed prior to analysis to reduce the influence of outliers. Statistical analyses were performed in $\mathrm{R}$ version 3.0.2 (R Development Core Team, 2011) using the Vegan R package version 2.0-6 (Oksanen et al., 2012).

\section{Results}

\subsection{Limnological variables}

The concentrations of Chl- $a$ (COR and FUN), TSS (FUN and PCO), TN (COR and ITU), and TP (FUN and MAR) presented seasonal differences (ANOVA, $p<0.05$ ). In contrast, $\mathrm{O}_{2}$ and conductivity did not show any temporal differences. The $\mathrm{pH}$ values fluctuated markedly (ANOVA, $p<0.05)$ in the waters of the LBC reservoir, which presented increased alkalinity during the rainy season ( $\mathrm{pH} 7.1$ to 7.4). Except for in MSM, the temperature showed a clear seasonal fluctuation (ANOVA, $p<0.05$ ) in the reservoirs, with the higher values always being recorded in the rainy season.
Overall, the temperature in the reservoirs did not exceed $30^{\circ} \mathrm{C}$. Finally, the reservoirs were classified as mesotrophic, eutrophic, and supereutrophic according to the TSI. In addition, there were seasonal differences in the trophic condition only in the FUN and MAR reservoirs (Table 3).

\subsection{Phytoplankton community}

There was no change in the phytoplankton abundance between the rainy and dry periods for all reservoirs (ANOVA, $p>0.05$ ) (Figure 2a). Cyanobacteria were the predominant phytoplankton in most of the reservoirs, except for the LBC reservoir, where Bacillariophyceae and Cyanobacteria were equally abundant in the rainy season, and Chlorophyceae, Bacillariophyceae, and other groups were predominant in the dry season. Other groups and Cyanobacteria were predominant in the dry season in the FUR reservoir. Finally, in the PCO reservoir, Bacillariophyceae were the predominant phytoplankton in the dry season (Figure $2 b$ ).

\subsection{Zooplankton community}

We identified a total of 99 species in this study, and rotifers were the richest group ( 62 species), followed by Cladocera (27 species) and Copepoda (10 species). The most common taxa of zooplankton in reservoirs were: rotifers, Keratella cochlearis (Gosse, 1851), Conochilus unicornis Rousselet, 1892, Hexarthra mira (Hudson, 1871) and Polyarthra vulgaris (Carlin, 1943); cladocerans, Bosmina hagmanni Stingelin, 1904, Bosminopsis deitersi Richard, 1895, Ceriodaphnia cornuta Sars, 1886, Ceriodaphnia silvestrii Dadayi, 1902, Daphnia gessneri Herbst, 1967, Diaphanosoma spinulosum Herbst, 1975 and Moina minuta Hansen, 1899; copepods, juvenile forms (nauplii and copepodites) and the species Thermocyclops decipiens (Kiefer, 1929) (Table 4).

Cladocerans and copepods (especially nauplii and copepodites) were the most abundant zooplankton groups in all of the reservoirs (Figure 3); the exception was MAR, which presented a predominance of rotifers in the rainy season (Figure 3a). Only the abundance of microcrustaceans was significantly different (ANOVA, $p<0.05$ ) between the rainy and dry seasons. The highest average values were always recorded in the dry period, and were observed for Cladocera in the ITU reservoir (Figure 3b); Cyclopoida in the COR, ITU, and PCO reservoirs (Figure 3c); and Calanoida in the COR and ITU reservoirs (Figure $3 \mathrm{~d}$ ).

Table 2. Classification of trophic states modified by Lamparelli (2004) for reservoirs.

\begin{tabular}{|c|c|c|c|}
\hline Trophic State & Criteria & $\mathrm{TP}\left(\mathrm{mg} \mathrm{m}^{-3}\right)$ & Chl-a (mg m $\left.{ }^{-3}\right)$ \\
\hline Ultraoligotrophic & TSI $<47$ & $\mathrm{TP} \leq 8$ & Chl-a $\leq 1.17$ \\
\hline Oligotrophic & $47<$ TSI $<52$ & $8<\mathrm{TP} \leq 19$ & $1.17<\mathrm{Chl}-\mathrm{a} \leq 3.24$ \\
\hline Mesotrophic & $52<$ TSI $<59$ & $19<\mathrm{TP} \leq 52$ & $3.24<$ Chl-a $\leq 11.03$ \\
\hline Eutrophic & $59<$ TSI $<63$ & $52<\mathrm{TP} \leq 120$ & $11.03<\mathrm{Chl}-\mathrm{a} \leq 30.55$ \\
\hline Supereutrophic & $63<$ TSI $<67$ & $120<\mathrm{TP} \leq 233$ & $30.55<$ Chl-a 69.05 \\
\hline Hypereutrophic & $\mathrm{TSI}>67$ & $233<\mathrm{TP}$ & $69.0<\mathrm{Chl}-\mathrm{a}$ \\
\hline
\end{tabular}

Abbreviations: Chl $a=$ chlorophyll $a, \mathrm{TP}=$ total phosphorus, $\mathrm{TSI}=$ Trophic State Index. 


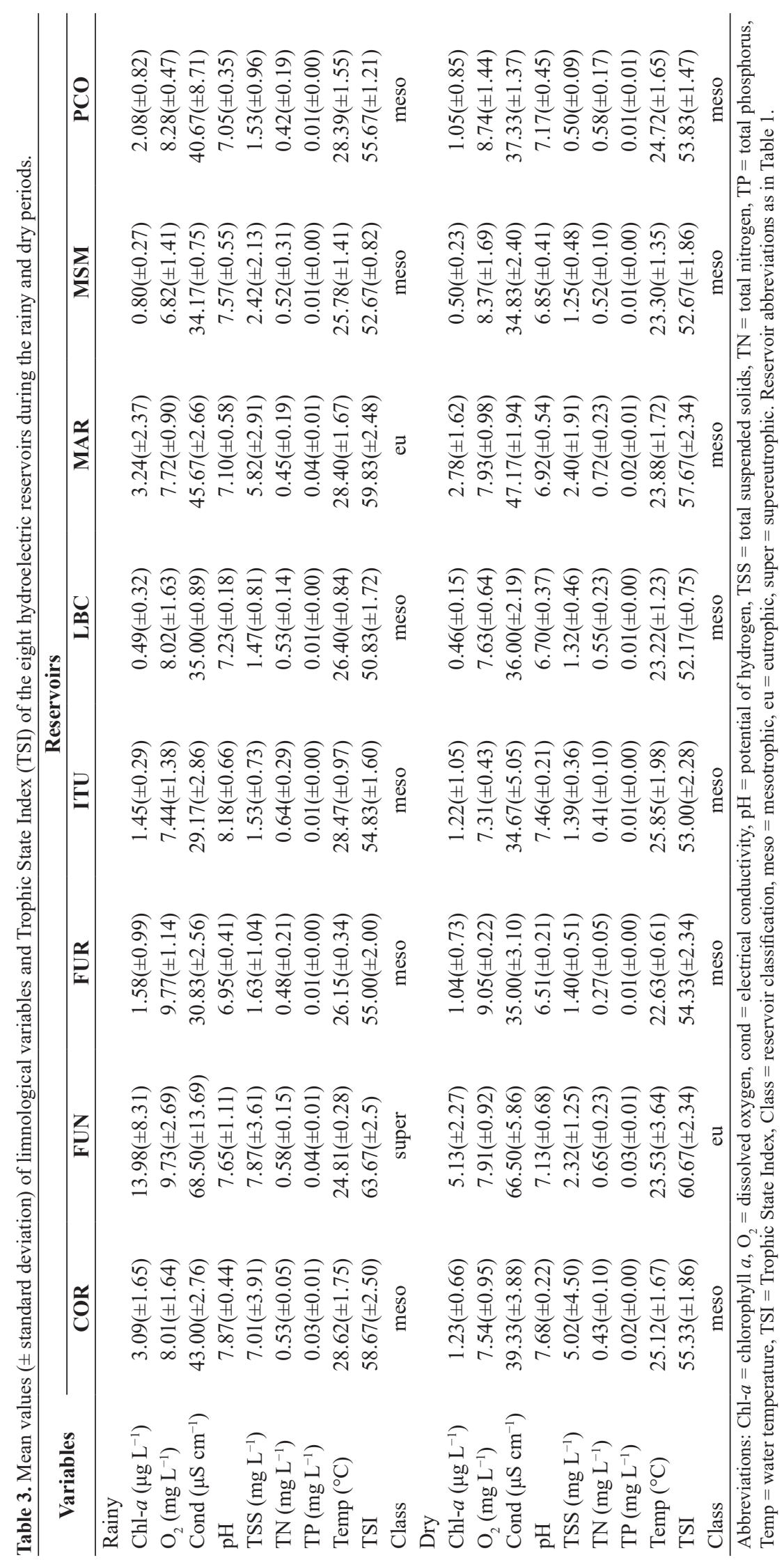




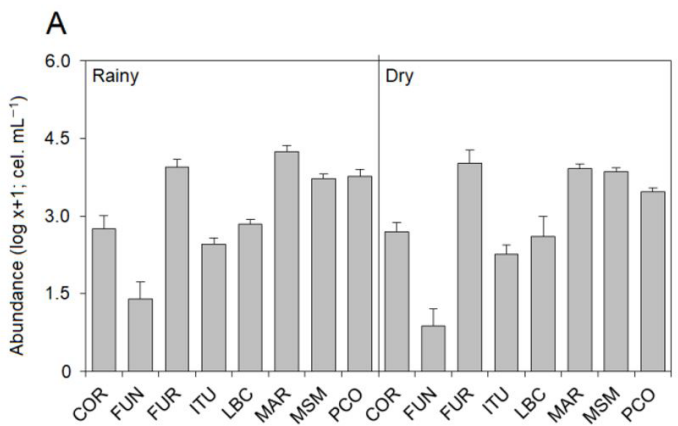

Reservoirs

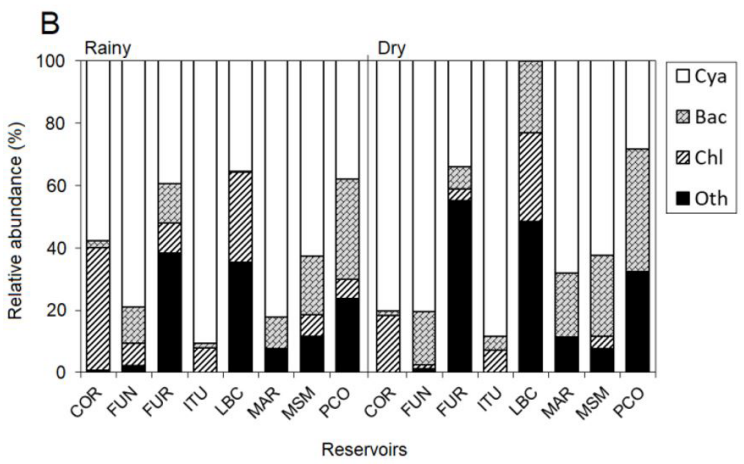

Reservoirs

Figure 2. Mean values ( \pm standard error) of phytoplankton abundance $(A)$ and relative abundance $(\%)(B)$ of the different phytoplankton taxonomic classes in the eight hydroelectric reservoirs during the rainy and dry periods. Abbreviations: Cya $=$ Cyanobacteria, $\mathrm{Bac}=$ Bacillariophyceae, $\mathrm{Chl}=$ Chlorophyceae, Oth $=$ Others. Reservoir abbreviations as in Table 1.

Table 4. Composition and mean abundance (ind. $\mathrm{m}^{-3}$ ) of zooplankton in the eight reservoirs.

\begin{tabular}{|c|c|c|c|c|c|c|c|c|}
\hline \multirow{3}{*}{ Taxa } & \multicolumn{8}{|c|}{ Reservoirs } \\
\hline & COR & FUN & FUR & ITU & LBC & MAR & MSM & PCO \\
\hline & \multicolumn{8}{|c|}{ Rainy / Dry } \\
\hline \multicolumn{9}{|l|}{ ROTIFERA } \\
\hline Anuraeopsis fissa Gosse, 1851 & & & & & & & $\bullet /$ & \\
\hline Ascomorpha ecaudis Perty, 1850 & $\bullet$ & $/ \bullet$ & $\bullet$ & & $/ \bullet$ & $\bullet$ & & $\bullet$ \\
\hline Ascomorpha ovalis (Bergendahl, 1892) & $\bullet /$ & & $\bullet$ & & $\bullet$ & & $\bullet$ & \\
\hline Asplanchna sieboldii (Leydig, 1854) & & & $\bullet /$ & & & $/ \bullet$ & $\bullet$ & \\
\hline Brachionus calyciflorus Pallas, 1776 & & $\bullet /$ & & & & o/ & & $\bullet /$ \\
\hline Brachionus caudatus Barrois and Daday, 1894 & $/ \bullet$ & & & & & & & \\
\hline Brachionus dolabratus Harring, 1914 & $/ \bullet$ & $\bullet /$ & $\bullet /$ & & $\bullet$ & $\bullet /$ & $\bullet /$ & $\bullet /$ \\
\hline Brachionus falcatus Zacharias, 1898 & $/ \bullet$ & & $\bullet /$ & & & $/ \bullet$ & $\bullet /$ & \\
\hline Brachionus forficula Wierzejski, 1891 & & & & & & $\bullet /$ & & \\
\hline Cephalodella sp. & $\bullet /$ & & & & & & & \\
\hline Collotheca sp. & $\bullet \bullet$ & $/ \bullet$ & & & $\bullet$ & & $/ \bullet$ & \\
\hline Conochilus coenobasis (Skorikow, 1914) & $\bullet \bullet$ & $\bullet \bullet$ & $\bullet / 0$ & & $\bullet$ & $\bullet \bullet$ & $\bullet \bullet$ & $\bullet \bullet$ \\
\hline Conochilus dossuarius Hudson, 1885 & & & & & & & $/ \bullet$ & \\
\hline Conochilus unicornis Rousselet, 1892 & $\bullet$ & ০/• & $\bullet \bullet$ & $\bullet / \bullet$ & $\bullet$ & ০/• & $\bullet$ & $\bullet /$ \\
\hline Dissotrocha sp. & & & & & & & & $\bullet /$ \\
\hline Euchlanis dilatata Ehrenberg, 1832 & & $\bullet$ & 10 & & & $\bullet$ & $/ \bullet$ & $\bullet /$ \\
\hline Filinia longiseta (Ehrenberg, 1834) & $\bullet \bullet$ & $\bullet$ & & & & & $/ \bullet$ & \\
\hline Filinia opoliensis (Zacharias, 1898) & & $\bullet /$ & $\bullet / \bullet$ & & $\bullet$ & $\bullet /$ & $\bullet$ & \\
\hline Filinia terminalis (Plate, 1886) & $\bullet \bullet$ & $\bullet$ & $\bullet /$ & & & & $\bullet /$ & \\
\hline Floscularia sp. & & & $\bullet /$ & & & & & \\
\hline Hexarthra intermedia Wiszniewski, 1929 & $\bullet /$ & & & & & & & $\bullet$ \\
\hline Hexarthra mira (Hudson, 1871) & $\bullet /$ & $/ \bullet$ & $\bullet /$ & $\bullet /$ & $\bullet$ & $\bullet /$ & $\bullet$ & $\bullet$ \\
\hline Hexarthra sp. & $\bullet /$ & & & & & & & \\
\hline Kellicottia bostoniensis (Rousselet, 1908) & $\bullet / \bullet$ & $\bullet / \bullet$ & $\bullet / \bullet$ & $/ \bullet$ & $/ \bullet$ & & $\bullet$ & $/ \bullet$ \\
\hline Keratella americana Carlin, 1943 & $\bullet \bullet$ & $\bullet /$ & $\bullet / 0$ & $\bullet$ & $\bullet /$ & $\bullet /$ & $\bullet \bullet$ & \\
\hline Keratella cochlearis (Gosse, 1851) & $\bullet \bullet$ & $\bullet \bullet$ & $\bullet \bullet$ & $\bullet$ & $\bullet$ & ○/• & $\bullet \bullet$ & $\bullet /$ \\
\hline Keratella lenzi (Hauer, 1953) & & & & $\bullet /$ & & ०/• & & \\
\hline Keratella tropica (Apstein, 1907) & $\bullet \bullet$ & $\bullet /$ & $\bullet \bullet$ & & $\bullet /$ & & $\bullet /$ & \\
\hline Lacinularia elliptica Shephard, 1897 & & $\bullet / 0$ & $\bullet / 0$ & & $\bullet$ & $\bullet / \bullet$ & ०/• & o/ \\
\hline Lecane bulla (Gosse, 1851) & $\bullet / \bullet$ & & & & $/ \bullet$ & & $\bullet /$ & $\bullet /$ \\
\hline Lecane cornuta (Müller, 1786) & $/ \bullet$ & & & & & & & \\
\hline Lecane ludwigi (Eckstein, 1883) & $\bullet \bullet$ & $1 \bullet$ & & & & & & \\
\hline
\end{tabular}

Symbols indicate men values during rainy (left of the slash) and dry (right of the slash) periods $\left(\bullet=>150\right.$ ind. $\mathrm{m}^{-3} ; \circ=<150$ to $>50$ ind. $\mathrm{m}^{-3}$; $\bullet=<50$ ind. $\mathrm{m}^{-3}$; no symbol $=$ absent). Reservoir abbreviations as in Table 1. 
Table 4. Continued...

\begin{tabular}{|c|c|c|c|c|c|c|c|c|}
\hline \multirow{3}{*}{ Taxa } & \multicolumn{8}{|c|}{ Reservoirs } \\
\hline & COR & FUN & FUR & ITU & LBC & MAR & MSM & PCO \\
\hline & \multicolumn{8}{|c|}{ Rainy / Dry } \\
\hline Lecane luna (Müller, 1776) & $\bullet$ & & & & $\bullet /$ & & $\bullet \bullet$ & $\bullet /$ \\
\hline Lecane lunaris (Ehrenberg, 1832) & $/ \bullet$ & $\bullet /$ & & & & & & \\
\hline Lecane monostyla (Daday, 1897) & & & & & & & $\bullet /$ & \\
\hline Lecane proiecta Hauer, 1956 & & & $\bullet /$ & & & $\bullet /$ & & \\
\hline Lecane signifera (Jennings, 1896) & & & & & $/ \bullet$ & & & \\
\hline Lecane stenroosi (Meissner, 1908) & & & & & $/ \bullet$ & & & \\
\hline Lepadella ovalis (Müller, 1786) & & & & & & & $\bullet /$ & \\
\hline Lepadella patella (Müller, 1773) & & & & & & & $\bullet /$ & \\
\hline Mytilina macrocera (Jennings, 1894) & & & & & & & $\bullet /$ & \\
\hline Notommata sp. & & & & & & & $\bullet /$ & \\
\hline Plationus patulus (Müller, 1786) & $\bullet$ & $\bullet /$ & & & & & $\bullet /$ & \\
\hline Platyias quadricornis (Ehrenberg, 1832) & & & & & $\bullet /$ & & $\bullet /$ & \\
\hline Ploesoma hudsoni (Imhof, 1891) & & & & & $/ \bullet$ & $\bullet \bullet$ & & \\
\hline Ploesoma truncatum (Levander, 1894) & & & & $\bullet /$ & & & & \\
\hline Polyarthra dolichoptera Idelson, 1925 & $\bullet /$ & & $\bullet /$ & & & & & \\
\hline Polyarthra vulgaris (Carlin, 1943) & $\bullet$ & $\bullet /$ & $\bullet / 0$ & $\bullet /$ & $\bullet$ & ০/० & $\bullet \bullet$ & $/ \bullet$ \\
\hline Pompholyx triloba Pejler, 1957 & & & $/ \bullet$ & & & & $\bullet /$ & \\
\hline Ptygura sp. & $\bullet \bullet$ & & $\bullet$ & & $\bullet / \bullet$ & & ०/• & $/ \bullet$ \\
\hline Sinantherina ariprepes Edmondson, 1939 & & $\bullet \bullet$ & ০/• & & $\bullet /$ & o/• & $\bullet /$ & $/ \bullet$ \\
\hline Stephanoceros fimbriatus (Goldfusz, 1820) & & & & & ০/० & & $\bullet \bullet$ & $\bullet$ \\
\hline Synchaeta stylata Wierzejski, 1893 & ০/० & $/ \bullet$ & $\bullet$ & $\bullet /$ & & ০/• & $\bullet \bullet$ & $\bullet \bullet$ \\
\hline Testudinella mucronata (Gosse, 1886) & & & & & & & $\bullet /$ & \\
\hline Testudinella patina (Hermann, 1783) & $/ \bullet$ & & & & & & $\bullet /$ & $\bullet /$ \\
\hline Trichocerca bidens (Lucks, 1912) & $\bullet /$ & & & & & & & \\
\hline Trichocerca cylindrica (Imhof, 1891) & $\bullet$ & & $\bullet / 0$ & & $\bullet \bullet$ & $\bullet \bullet$ & $\bullet \bullet$ & $\bullet$ \\
\hline Trichocerca insignis (Herrick, 1885) & $\bullet /$ & & & & & & & $\bullet$ \\
\hline Trichocerca insulana (Hauer, 1937) & $/ \bullet$ & & & & & & & \\
\hline Trichocerca similis (Wierzejski, 1893) & & & & & & & $\bullet /$ & \\
\hline Trichocerca sp. & & & & & & & $/ \bullet$ & \\
\hline Trichotria tetractis (Ehrenberg, 1830) & $/ \bullet$ & & & & & & $/ \bullet$ & \\
\hline CLADOCERA & & & & & & & & \\
\hline Alona guttata Sars, 1862 & & $/ \bullet$ & & & & & & \\
\hline Alonella dadayi Birge, 1910 & $/ \bullet$ & & 10 & & & & $\bullet /$ & \\
\hline Bosmina freyi Melo and Hebert, 1994 & & & & & o/ & ০/० & $/ \bullet$ & \\
\hline Bosmina hagmanni Stingelin, 1904 & $\bullet \bullet$ & $\bullet / 0$ & ০/• & $\bullet / 0$ & $\bullet / 0$ & $\bullet / \circ$ & $\bullet / 0$ & $\bullet / 0$ \\
\hline Bosmina longirostris (Müller, 1785) & & & & $/ \bullet$ & & & & \\
\hline Bosminopsis deitersi Richard, 1895 & $\bullet$ & $\bullet \bullet$ & $\bullet \bullet$ & $\bullet \bullet$ & $\bullet$ & ০/• & $\bullet$ & $\bullet \bullet$ \\
\hline Camptocercus australis Sars, 1896 & & & & & $/ \bullet$ & & & \\
\hline Ceriodaphnia cornuta Sars, 1886 & $\bullet$ & $\bullet / 0$ & ০/• & $\bullet / 0$ & $\bullet / \bullet$ & $\bullet / \bullet$ & ०/० & $\bullet / \bullet$ \\
\hline Ceriodaphnia silvestrii Dadayi, 1902 & $\bullet / 0$ & $\bullet / 0$ & $\bullet / \bullet$ & $\bullet \bullet$ & $\bullet / \bullet$ & $\bullet / \bullet$ & ০/० & $\bullet / \bullet$ \\
\hline Coronatella poppei (Richard, 1897) & & & & & $/ \bullet$ & $\bullet \bullet$ & & \\
\hline Daphnia gessneri Herbst, 1967 & $\bullet$ & $\bullet / \bullet$ & $\bullet \bullet$ & $\bullet / 0$ & $\bullet / 0$ & $\bullet / \bullet$ & $\bullet / 0$ & ০/• \\
\hline Diaphanosoma birgei Korineck, 1981 & $\bullet \bullet$ & & $\bullet$ & $\bullet \bullet$ & $\bullet / 0$ & & $\bullet /$ & $/ \bullet$ \\
\hline Diaphanosoma brevireme Sars, 1901 & & & & $\bullet / 0$ & $\bullet / 0$ & $\bullet \bullet$ & & ○/• \\
\hline Diaphanosoma polyspina Korovchinsky, 1982 & & & & & $\bullet /$ & & & \\
\hline Diaphanosoma sp. & & & & $\bullet \bullet$ & & & & \\
\hline Diaphanosoma spinulosum Herbst, 1975 & ০/• & $\bullet / \circ$ & $\bullet / \bullet$ & $\bullet$ & ○/• & ○/• & $\bullet / \bullet$ & $\bullet / \circ$ \\
\hline Disparalona leptorhyncha Smirnov, 1996 & $/ \bullet$ & & & & & & & \\
\hline Ilyocryptus spinifer Herrick, 1882 & $/ \bullet$ & & $\bullet /$ & & & $\bullet /$ & & \\
\hline Leberis davidi (Richard, 1895) & $/ \bullet$ & & & & & & & \\
\hline
\end{tabular}

Symbols indicate men values during rainy (left of the slash) and dry (right of the slash) periods $\left(\bullet=>150\right.$ ind. $\mathrm{m}^{-3} ; \circ=<150$ to $>50$ ind. $\mathrm{m}^{-3}$; $\cdot=<50$ ind. $\mathrm{m}^{-3}$; no symbol $=$ absent). Reservoir abbreviations as in Table 1. 
Table 4. Continued...

\begin{tabular}{|c|c|c|c|c|c|c|c|c|}
\hline \multirow{3}{*}{ Taxa } & \multicolumn{8}{|c|}{ Reservoirs } \\
\hline & COR & FUN & FUR & ITU & LBC & MAR & MSM & PCO \\
\hline & \multicolumn{8}{|c|}{ Rainy / Dry } \\
\hline Leydigia ipojucae Brehm, 1938 & & $\%$ & & & & & & \\
\hline Leydigia schubarti Brehm and Thomsen, 1936 & & $\bullet /$ & & & & & & \\
\hline Macrothrix laticornis (Jurine, 1820) & & & & & & & $\bullet /$ & \\
\hline Moina micrura Kurz, 1874 & & & $\bullet / 0$ & & & & & \\
\hline Moina minuta Hansen, 1899 & $\bullet$ & $\bullet /$ & $\bullet / \bullet$ & $/ \bullet$ & $\bullet / \bullet$ & $\bullet / 0$ & ०/० & $\bullet$ \\
\hline Ovalona glabra (Sars, 1901) & & & & & & $/ \bullet$ & & \\
\hline Simocephalus serrulatus (Koch, 1841) & & & & $/ \bullet$ & & & & \\
\hline Simocephalus sp. & $\bullet$ & & $/ \bullet$ & & & & & \\
\hline COPEPODA & & & & & & & & \\
\hline CYCLOPOIDA & & & & & & & & \\
\hline Nauplii & ০/• & $\bullet / \bullet$ & $\bullet / \bullet$ & ০/• & $\bullet / \bullet$ & $\bullet / \bullet$ & $\bullet / \bullet$ & ০/• \\
\hline Copepodite & ০/• & $\bullet / \bullet$ & $\bullet / \bullet$ & ০/• & $\bullet / \bullet$ & $\bullet / \bullet$ & $\bullet / \bullet$ & $\bullet / \bullet$ \\
\hline Mesocyclops meridianus (Kiefer, 1926) & & $\bullet \bullet$ & & & $\bullet /$ & $/ \bullet$ & $\bullet /$ & $\bullet$ \\
\hline Thermocyclops decipiens (Kiefer, 1929) & $\bullet / 0$ & $\bullet / \bullet$ & $\bullet / \bullet$ & $/ \bullet$ & $\bullet \bullet$ & $\bullet \bullet$ & $\bullet \bullet$ & $\bullet / 0$ \\
\hline Thermocyclops minutus (Lowndes, 1934) & $/ \bullet$ & $\bullet \bullet$ & $\bullet \bullet$ & & $\bullet / 0$ & $\bullet$ & $\bullet$ & $\bullet$ \\
\hline CALANOIDA & & & & & & & & \\
\hline Nauplii & $\bullet / 0$ & $\bullet \bullet$ & ০/• & $\bullet \bullet$ & $\bullet / 0$ & $\bullet / \bullet$ & $\bullet / 0$ & ০/• \\
\hline Copepodite & $\bullet / \bullet$ & $\bullet / \bullet$ & $\bullet / \bullet$ & ০/• & $\bullet / \bullet$ & $\bullet / \bullet$ & $\bullet / \bullet$ & $\bullet / \bullet$ \\
\hline Argyrodiaptomus azevedoi (Wright, 1935) & $\bullet$ & $\bullet \bullet$ & & $/ \bullet$ & & & & \\
\hline Argyrodiaptomus furcatus (Sars, 1901) & & & & $/ \bullet$ & & & & \\
\hline Argyrodiaptomus sp. & & & & $\bullet / 0$ & & & & \\
\hline Notodiaptomus cearensis (Wright, 1936) & $\bullet / 0$ & $\bullet / 0$ & ০/• & $\bullet \bullet$ & & & $\bullet /$ & \\
\hline Notodiaptomus henseni (Dahl, 1894) & & $\bullet \bullet$ & & $\bullet \bullet$ & $\bullet / \bullet$ & $\bullet / \bullet$ & $\bullet / 0$ & $\bullet / \bullet$ \\
\hline Notodiaptomus iheringi (Wright, 1935) & & & & & $\bullet / \bullet$ & $\bullet / \bullet$ & & \\
\hline Notodiaptomus sp. & & & & $\bullet$ & & & & \\
\hline
\end{tabular}
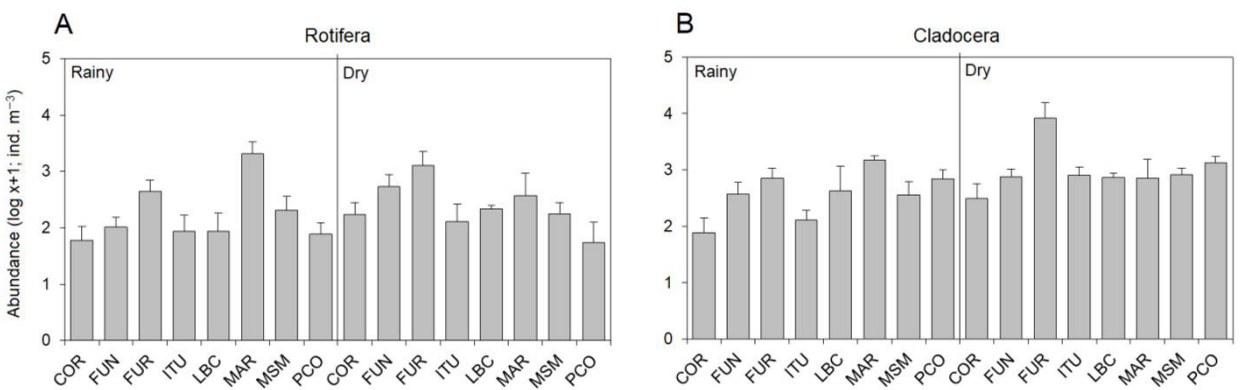

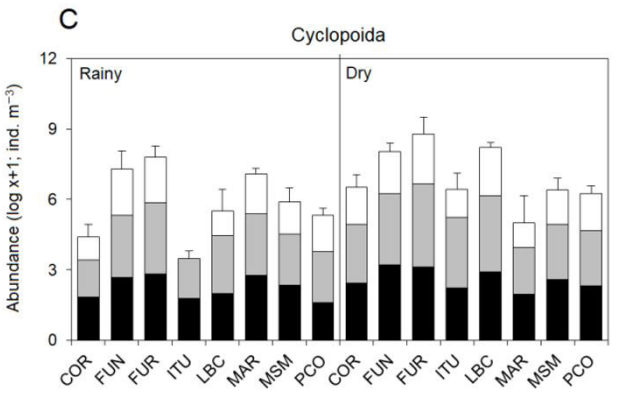

Reservoirs

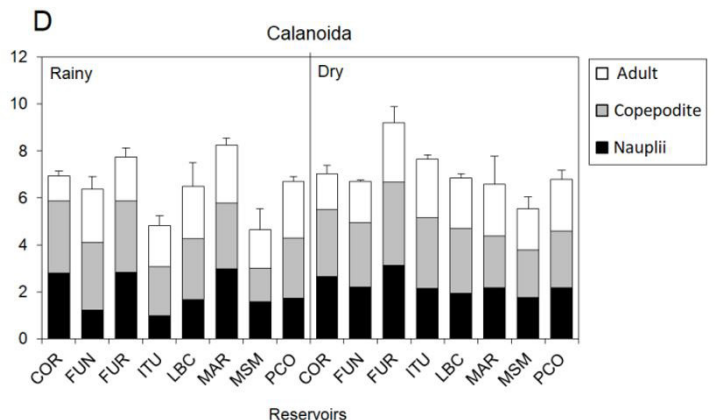

Figure 3. Mean values ( \pm standard error) of abundance of Rotifera (A), Cladocera (B), and copepods of Cyclopoida (C) and Calanoida (D) in its different developmental phases, in the eight hydroelectric reservoirs during the rainy and dry periods. Bars $=$ standard errors. Reservoir abbreviations as in Table 1. 

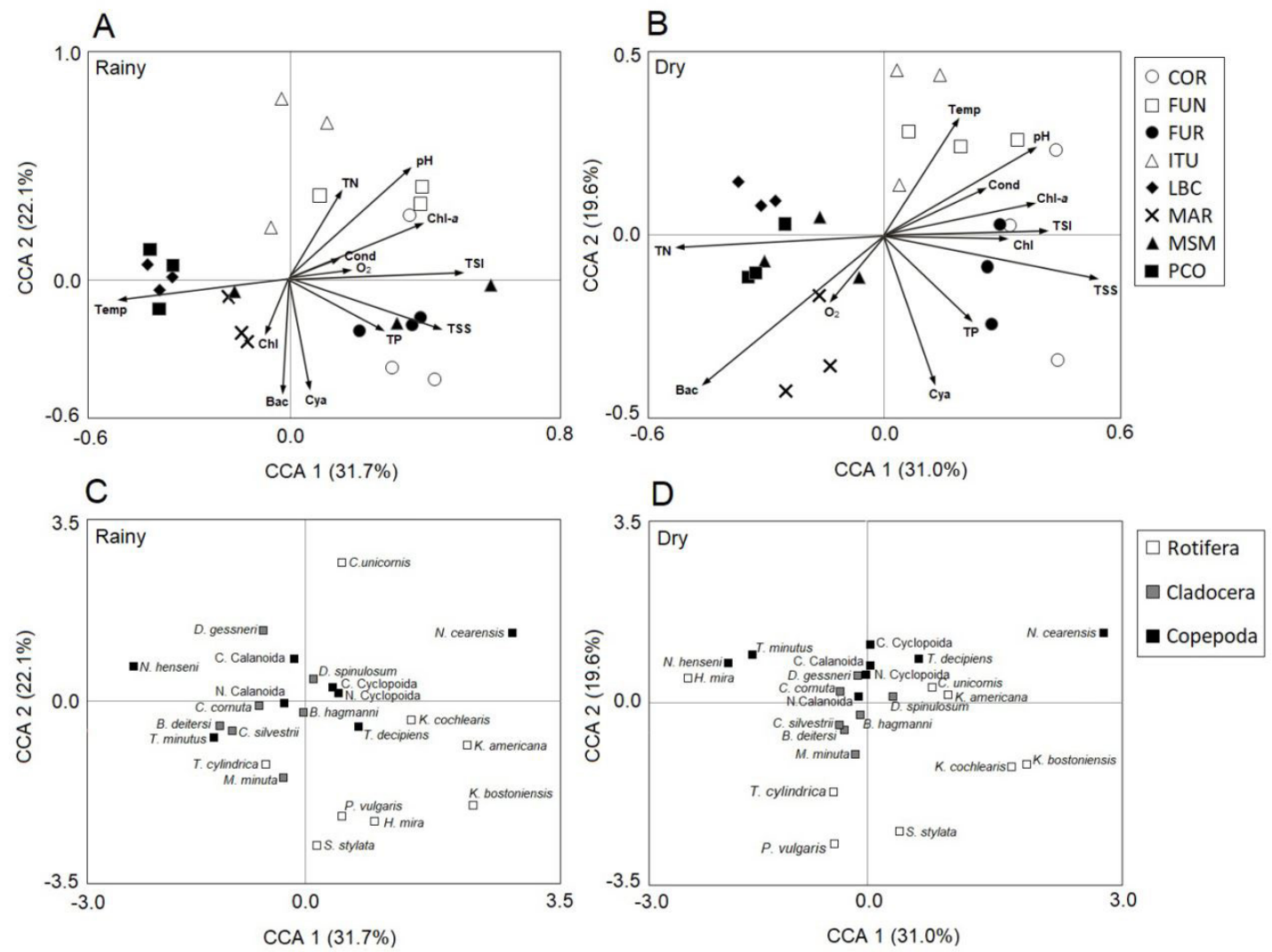

Figure 4. The canonical correspondence analysis (CCA) shows the relationships among the environmental variables and environments of the reservoirs $(\mathrm{A}, \mathrm{B})$ and zooplankton taxa $(\mathrm{C}, \mathrm{D})$ during the rainy and dry periods. Abbreviations: Cya $=$ Cyanobacteria, $\mathrm{Bac}=$ Bacillariophyceae, $\mathrm{Chl}=$ Chlorophyceae, $\mathrm{Chl}-a=$ chlorophyll $a, \mathrm{O}_{2}=$ dissolved oxygen, cond $=$ electrical conductivity, $\mathrm{TSS}=$ total suspended solids, $\mathrm{TN}=$ total nitrogen, $\mathrm{TP}=$ total phosphorus, Temp $=$ water temperature, TSI $=$ Trophic State Index. Reservoir abbreviations as in Table 1.

\subsection{Relationships between the zooplankton community dynamics and environmental variables}

According to the CCAs, the abundance of zooplankton was correlated with the environmental variables, as well as with the reservoirs in both of the analysed hydrological periods (Permutest, $p=0.001$ ). Furthermore, the analysis results illustrated that the environmental variables influenced $53.9 \%$ and $50.6 \%$ of the variations in abundance during the rainy and dry periods, respectively. Some species had an association with environmental variables during the analysed periods. The correlations for the rainy period are as follows: D. gessneri and Notodiaptomus henseni (Dahl, 1894) were negatively correlated with the TSS and TP concentrations, and Cyanobacteria abundance; B. deitersi, C. silvestrii, and Thermocyclops minutus (Lowdes, 1934) were positively correlated with the temperature and Bacillariophyta abundance; C. unicornis and Notodiaptomus cearensis (Wright, 1953) were positively correlated with the TSI, pH, and Chl- $a$ concentrations; and Kellicottia bostoniensis (Rousselet, 1908), K. cochlearis, H. mira, and $P$. vulgaris were positively correlated with the TSS and TP concentrations (Figure $4 \mathrm{a}, 4 \mathrm{c}$ ). The correlations for the dry period are as follows: H. mira, T. minutus, and $N$. hensen $i$ were negatively correlated with the TSS and TP concentrations and Cyanobacteria abundances; Trichocerca cylindrica (Imhof, 1891), P. vulgaris, and M. minuta were positively correlated with the TN concentration and Bacillariophyta abundance; Keratella americana Carlin, 1943, T. decipiens, and N. cearensis were positively correlated with the TSI, $\mathrm{pH}$, and Chl- $a$ concentrations; and K. bostoniensis, K. cochlearis, and Synchaeta stylata Wierzejski, 1893 were positively correlated with the TSS concentration (Figure 4b, 4d).

\section{Discussion}

Most of the reservoirs studied exhibited mesotrophic conditions, with the exception of the FUN and MAR reservoirs, which were experiencing eutrophication at the time of study. These reservoirs differ in many morphofunctional parameters, such as size, water residence time (WRT), and depth. Thus, both the trophic variation and morphofunctional characteristics of the reservoirs can affect zooplankton population dynamics differently (Perbiche-Neves et al., 2013). 
Rotifers were the organisms that most contributed to the total species richness of the zooplankton. The high diversity of this group in reservoirs has been a recurring pattern in Brazil and is mainly attributed to the opportunistic characteristics of this group (e.g., wide food spectrum, high population turnover) (Takahashi et al., 2009; De-Carli et al., 2018; Picapedra et al., 2020). In turn, great occurrences and abundances of cladocerans (especially B. hagmanni and $C$. cornuta) and juvenile copepods can be an important indicator of the beginning of the eutrophication process in these reservoirs. Although most reservoirs are predominantly mesotrophic, the predominance of Cyanobacteria indicates the enrichment of nutrients in these systems. Higher concentrations of detritus and nutrients favour the growth of bacteria and protozoa, an important source of food for small filter feeders such as nauplii and small cladocerans (e.g., bosminids) (Brito et al., 2011). In addition, the great contribution of juvenile stages of copepods in relation to adults is often found in Brazilian reservoirs. The production of a large number of larval stages can be considered a reproductive strategy of this group to compensate for high mortality before they reach the final stage (Bonecker et al., 2001; Lansac-Tôha et al., 2005; De-Carli et al., 2018).

In this study, seasonal changes in abundance were observed for microcrustaceans (cladocerans and copepods) in the COR, ITU, and PCO reservoirs, with lower values in the rainy season. These decreases in abundance may have resulted from the dilution effect of a higher volume of rainwater, and partially by the removal of these populations at the outlets downstream of these reservoirs (Gazonato-Neto et al., 2014). Some authors (Bonecker et al., 2001; Takahashi et al., 2009) also observed a lower abundance of microcrustaceans during the rainy period in the COR reservoir.

The use of zooplankton species as biological indicators can provide important information on current and past processes, such as changes in biological relationships and in the physical and chemical properties of water (Perbiche-Neves et al., 2019). In this study, the TP and TSS concentrations and phytoplankton abundance were most associated with the fluctuation of zooplankton in the reservoirs. For example, the cladoceran D. gessneri and the copepod $N$. hensen $i$ were abundant during the rainy and dry periods in the LBC and PCO reservoirs, which had lower TSS concentrations and Cyanobacteria abundances. For some species of zooplankton, the food efficiency may decrease when food is mixed with suspended particles, even if phytoplankton are abundant in the water (Arruda et al., 1983).

The suspension of matter can have a negative effect on zooplankton, causing mechanical disturbances (obstruction and/or clogging) within the filtering apparatus, reducing the feeding and growth rates of these organisms and compromising important biological interactions (Claps et al., 2011; José de Paggi and Paggi, 2014). In addition, cyanobacterial filaments or mucilages can also interfere with the filtration apparatus of large cladocerans and calanoid copepods, which leads to the decline and replacement of these populations by small rotifers (Sendacz et al., 2006; Eskinazi-Sant'Anna et al., 2013), as observed in the FUR and COR reservoirs.

In the MAR reservoir, the variation in the abundance of some species of cladocerans (e.g., B. hagmanni, M. minuta, and $B$. deitersi) and the rotifer $T$. cylindrica were associated with the abundance of diatoms (Bacillariophyceae) during the rainy and dry periods. According to Eskinazi-Sant'Anna et al. (2013), diatoms algae are more palatable and nutritious than Cyanobacteria, and possibly favoured the development of these populations. In contrast, it was observed that the rotifers K. bostoniensis (invasive species), Keratella spp., H. mira, and P. vulgaris occurred in environments with a high abundance of Cyanobacteria and high levels of TP and TSS in the FUR (rainy and dry periods), MSM (rainy period) and COR (rainy period) reservoirs, and C. unicornis occurred in eutrophic environments in the FUN (rainy and dry periods) reservoir. These species are typical of meso-eutrophic environments and feed on solid suspended particles and colloids derived from bacteria that decompose organic material (Branco et al., 2002; Sousa et al. 2008; De-Carli et al. 2018). However, even with the trend observed in this study, the use of rotifers as bioindicators must be performed with care, as contrasting results can be found in the literature. For example, Nogueira (2001) and Sampaio et al. (2002) found high frequencies of C. unicornis, K. americana, K. cochlearis, and P. vulgaris in oligotrophic reservoirs on the Paranapanema River.

In relation to copepods, the co-occurrence of $T$. minutus and $T$. decipiens, with a higher occurrence of $T$. decipiens, may indicate a transition between mesotrophic and eutrophic conditions in reservoirs. T. minutus is more frequent in oligotrophic waters, whereas it is replaced by T. decipiens in eutrophic waters (Nogueira et al., 2002; Perbiche-Neves et al., 2016). In mesotrophic lakes, both species would be found together or in a seasonally alternating pattern (Sartori et al., 2009; Serafim-Júnior et al., 2016). $T$. decipiens was the most abundant in six of the eight reservoirs studied, mainly during the rainy season when the highest TP and TSS concentrations were observed. Pinto-Coelho (1987), studying Paranoá Lake, considered that T. decipiens had a greater growth capacity in environments with the recurrent introduction of allochthonous materials. In turn, $N$. cearensis was the most constant in the COR, FUN, and FUR reservoirs. This species is normally linked to environments with high electrical conductivity and productivity, and can feed on Cyanobacteria (Bouvy et al., 2001; Matsumura-Tundisi and Galizia Tundisi, 2003; Sartori et al., 2009).

Based on our results, it can be concluded that most reservoirs are mesotrophic (COR, FUR, ITU, LBC, MSM and PCO) and some are undergoing eutrophication (FUN and MAR), mainly due to the entry of nutrients from the urban and agricultural areas in the bodies of these systems. Consequently, there was a predominance of Cyanobacteria in the reservoirs, with the exception of LBC. Seasonal changes in zooplankton abundance were found only for microcrustaceans in some reservoirs, with lower values during the rainy season, possibly due to a dilution 
effect caused by rain. Species such as K. bostoniensis, H. mira, Keratella spp., and P. vulgaris were indicators of environments with higher levels of nutrients and a predominance of Cyanobacteria (e.g., COR and FUR). Additionally, microcrustaceans such as $T$. decipiens, $N$. cearensis and juvenile forms (nauplii and copepodites) of copepods occurred in environments that presented higher TSI values of FUN and ITU reservoirs. In contrast, the microcrustaceans $D$. gessneri and $N$. henseni can be considered indicators of more desirable water quality conditions (e.g., PCO and LBC). This study provided relevant information on the water quality of Brazilian reservoirs.

\section{Acknowledgements}

The authors would like to thank the CAPES (Coordenação de Aperfeiçoamento de Pessoal de Nível Superior) for the post-doctorate fellowship granted to first author. We would also like to thank the Grupo de Pesquisas em Recursos Pesqueiros e Limnologia of the Universidade Estadual do Oeste do Paraná and FURNAS Centrais Elétricas S. A. for the logistic and financial support. We are also grateful to the anonymous reviewers, whose detailed comments and constructive suggestions improved the quality of the manuscript.

\section{References}

AGOSTINHO, A.A., GOMES, L.C. and PELICICE, F.M., 2007. Ecologia e manejo de recursos pesqueiros em reservatórios do Brasil. Maringá: EDUEM.

AMERICAN PUBLIC HEALTH ASSOCIATION - APHA, 2005. Standard methods for the examination water and wastewater. Washington: APHA.

ARRUDA, J.A., MARZOLF, G.R. and FAULK, R.T., 1983. The role of suspended sediments in the nutrition of zooplankton in turbid reservoirs. Ecology, vol. 64, no. 5, pp. 1225-1235. http:// dx.doi.org/10.2307/1937831.

BAUMGARTNER, M.T., BAUMGARTNER, G. and GOMES, L.C., 2017. The effects of rapid water level changes on fish assemblages: the case of a spillway gate collapse in a Neotropical reservoir. River Research and Applications, vol. 33, no. 4, pp. 548-557. http://dx.doi.org/10.1002/rra.3110.

BONECKER, C.C. and AOYAGUI, A.S.M., 2005. Relationships between rotifers, phytoplankton and bacterioplankton in the Corumbá Reservoir, Goiás State, Brazil. Hydrobiologia, vol. 546, no. 1, pp. 415-421. http://dx.doi.org/10.1007/s10750-005-4284-1.

BONECKER, C.C., LANSAC-TÔHA, F.A., VELHO, L.F.M. and ROSSA, D.C., 2001. The temporal distribution pattern of copepods in Corumbá Reservoir, State of Goiás, Brazil. Hydrobiologia, vol. 453-454, pp. 375-384. http://dx.doi.org/10.1023/A:1013142101266.

BOTTRELL, H.H., DUNCAN, A., GLIWICZ, Z.M., GRYGIEREK, E., HERZIG, A., HILLBRICHT-ILKOWSKA, A., KURASAWA, H., LARSSON, P. and WEGLENSKA, T., 1976. Review of some problems in zooplankton production studies. Netherlands Journal of Zoology, vol. 24, pp. 419-456.
BOUVY, M., PAGANO, M. and TROUSSELLIER, M., 2001. Effects of a cyanobacterial bloom (Cylindrospermopsis raciborskii) on bacteria and zooplankton communities in Ingazeira reservoir (northeast Brazil). Aquatic Microbial Ecology, vol. 25, pp. 215227. http://dx.doi.org/10.3354/ame025215.

BRANCO, C.W.C., ROCHA, M.-I.A., PINTO, G.F.S., GOMARA, G.A. and FILIPPO, R.D., 2002. Limnological features of Funil Reservoir (R.J., Brazil) and indicator properties of rotifers and cladocerans of the zooplankton community. Lakes and Reservoirs: Research and Management, vol. 7, no. 2, pp. 87-92. http://dx.doi. org/10.1046/j.1440-169X.2002.00177.x.

BRITO, S.L., MAIA-BARBOSA, P.M. and PINTO-COELHO, R.M., 2011. Zooplankton as an indicator of trophic conditions in two large reservoirs in Brazil. Lakes and Reservoirs: Research and Management, vol. 16, no. 4, pp. 253-264. http://dx.doi. org/10.1111/j.1440-1770.2011.00484.x.

BRITO, S.L., MAIA-BARBOSA, P. and PINTO-COELHO, R.M., 2016. Secondary productivity of main microcrustacean species of two tropical reservoirs in Brazil and its relationship with trophic state. Journal of Limnology, vol. 75, no. 2, pp. 320-329. http:// dx.doi.org/10.4081/jlimnol.2016.1267.

CLAPS, M.C., GABELLONE, N.A. and BENÍTEZ, H.H., 2011. Seasonal changes in the vertical distribution of rotifers in a eutrophic shallow lake with contrasting states of clear and turbid water. Zoological Studies (Taipei, Taiwan), vol. 50, no. 4, pp. 454-465.

DE-CARLI, B.P., ALBUQUERQUE, F.P., MOSCHINI-CARLOS, V. and POMPÊO, M., 2018. Comunidade zooplanctônica e sua relação com a qualidade da água em reservatórios do Estado de São Paulo. Iheringia. Série Zoologia, vol. 108, no. 0, pp. e2018013. http://dx.doi.org/10.1590/1678-4766e2018013.

DE-MOTT, W.R., GULATI, R.D. and VAN DONK, E., 2001. Daphnia food limitation in three hypertrophic Dutch lakes: evidence for exclusion of large bodied species by interfering filaments of cyanobacteria. Limnology and Oceanography, vol. 46, no. 8, pp. 2054-2060. http://dx.doi.org/10.4319/lo.2001.46.8.2054.

ELMOOR-LOUREIRO, L.M.A., 1997. Manual de identificação de cladóceros límnicos do Brasil. Brasília: Universa.

ESKINAZI-SANT'ANNA, E.M., MENEZES, R., COSTA, I.S., ARAÚJO, M., PANOSSO, R. and ATTAYDE, J.L., 2013. Zooplankton assemblages in eutrophic reservoirs of the Brazilian semi-arid. Brazilian Journal of Biology $=$ Revista Brasileira de Biologia, vol. 73, no. 1, pp. 37-52. http://dx.doi.org/10.1590/ S1519-69842013000100006. PMid:23644787.

GAZONATO-NETO, A.J., SILVA, L.C., SAGGIO, A.A. and ROCHA, O., 2014. Zooplankton communities as eutrophication bioindicators in tropical reservoirs. Biota Neotropica, vol. 14, no. 4, pp. e20140018. http://dx.doi.org/10.1590/1676-06032014001814.

JOSÉ DE PAGGI, S. and PAGGI, J.C., 2014. El zooplancton de los grandes ríos sudamericanos con planície de inundación. Revista FABICIB, vol. 18, pp. 166-194. http://dx.doi.org/10.14409/ fabicib.v18i0.4853.

KOSTE, W., 1978. Rotatoria Die Rädertiere Mitteleuropas begründet von Max Voigt - Monogononta. Berlin: Gebrüder Borntraeger.

LAMPARELLI, M.C., 2004. Grau de trofia em corpos d'água do estado de São Paulo: avaliação dos métodos de monitoramento. São Paulo: Universidade de São Paulo, 238 p. Tese de Doutorado em Ecologia.

LANSAC-TÔHA, F.A., BONECKER, C.C. and VELHO, L.F.M. 2005. Estrutura da comunidade zooplanctônica em reservatórios. 
In: L. RODRIGUES, S.M. THOMAZ, A.A. AGOSTINHO and L.C. GOMES, eds. Biocenoses em reservatórios: padrões espaciais e temporais. São Carlos: RiMa, pp. 115-127.

LEGENDRE, P., OKSANEN, J. and TER-BRAAK, C.J.F., 2011. Testing the significance of canonical axes in redundancy analysis. Methods in Ecology and Evolution, vol. 2, no. 3, pp. 269-277. http://dx.doi.org/10.1111/j.2041-210X.2010.00078.x.

LOKEN, L.C., CRAWFORD, J.T., DORNBLASER, M.M., STRIEGL, R.G., HOUSER, J.N., TURNER, P.A. and STANLEY, E.H., 2018. Limited nitrate retention capacity in the Upper Mississippi River. Environmental Research Letters, vol. 13, no. 7, pp. 074030. http://dx.doi.org/10.1088/1748-9326/aacd51.

LUND, J.W.G., KIPLING, C. and LECREN, E.D., 1958. The inverted microscope method of estimating algal number and the statistical basis of estimating by counting. Hydrobiologia, vol. 11, no. 2, pp. 143-170. http://dx.doi.org/10.1007/BF00007865.

MATSUMURA-TUNDISI, T. and GALIZIA TUNDISI, J., 2003. Calanoida (Copepoda) species composition changes in the reservoirs of São Paulo State (Brazil) in the last twenty years. Hydrobiologia, vol. 504, no. 1-3, pp. 215-222. http://dx.doi. org/10.1023/B:HYDR.0000008521.43711.35

MATSUMURA-TUNDISI, T., 1986. Latitudinal distribution of Calanoida copepods in freshwater aquatic systems of Brazil. Brazilian Journal of Biology $=$ Revista Brasileira de Biologia, vol. 46 , no. 3, pp. 527-553.

NOGUEIRA, M.G., 2001. Zooplankton composition, dominance and abundance as indicators of environmental compartmentalization in Jurumirim Reservoir (Paranapanema River), São Paulo, Brazil. Hydrobiologia, vol. 455, no. 1-3, pp. 1-18. http://dx.doi. org/10.1023/A:1011946708757.

NOGUEIRA, M.G., JORCIN, A., VIANNA, N.C. and BRITTO, Y.C.T., 2002. Uma avaliação dos processos de eutrofização nos reservatórios em cascata do Rio Paranapanema (SP/PR), Brasil. In: A.F. CIRELLI and G.C. MARQUISÁ, eds. El agua en Iberoamerica: de la Limnología a la Gestión en Sudamérica. Buenos Aires: Programa Iberoamericano de Ciencia y Tecnologia para el Desarrollo, pp. 91-106.

OKSANEN, J., BLANCHET, F.G. and KINDT, R. 2012 [viewed 1 June 2018]. Vegan: community ecology package: R package. Version 2.0-6 [online]. Vienna: R Foundation for Statistical Computing. Available from: http://vegan.r-forge.r-project.org/

PERBICHE-NEVES, G., FILETO, C., LAÇO-PORTINHO, J., TROGUER, A. and SERAFIM JUNIOR, M., 2013. Relations among planktonic rotifers, Cyclopoid copepods, and water quality in two Brazilian reservoirs. Latin American Journal of Aquatic Research, vol. 41, no. 1, pp. 138-149. http://dx.doi.org/10.3856/ vol41-issue1-fulltext-11.

PERBICHE-NEVES, G., SAITO, V.S., PREVIATTELLI, D., ROCHA, C.E.F. and NOGUEIRA, M.G., 2016. Cyclopoid copepods as bioindicators of eutrophication in reservoirs: do patterns hold for large spatial extents? Ecological Indicators, vol. 70, pp. 340-347. http://dx.doi.org/10.1016/j.ecolind.2016.06.028.

PERBICHE-NEVES, G., SAITO, V.S., SIMÕES, N.R., DEBASTIANIJÚNIOR, J.R., NALIATO, D.A.O. and NOGUEIRA, M.G., 2019. Distinct responses of Copepoda and Cladocera diversity to climatic, environmental, and geographic filters in the La Plata River basin. Hydrobiologia, vol. 826, no. 1, pp. 113-127. http:// dx.doi.org/10.1007/s10750-018-3722-9.
PICAPEDRA, P.H.S., FERNANDES, C., TABORDA, J., BAUMGARTNER, G., and SANCHES, P.V., 2020. A long-term study on zooplankton in two contrasting cascade reservoirs (Iguaçu River, Brazil): effects of inter-annual, seasonal, and environmental factors. PeerJ, vol. 8, pp. e8979. http://dx.doi. org/10.7717/peerj. 8979 .

PINTO-COELHO, R.M., 1987. Flutuações sazonais e de curta duração na comunidade zooplanctônica do Lago Paranoá, BrasíliaDF, Brasil. Brazilian Journal of Biology = Revista Brasileira de Biologia, vol. 47, no. 1-2, pp. 17-29.

R DEVELOPMENT CORE TEAM, 2011 [viewed 1 June 2018]. $R$ : a language and environment for statistical computing [online]. Vienna: R Foundation for Statistical Computing. Available from: http://vegan.r-forge.r-project.org/

REID, J.W., 1985. Key to the identification and a reference list for the continental South American species of free-living Cyclopoida (Crustacea, Copepoda). Boletim de Zoologia, vol. 9, no. 9, pp. 17. http://dx.doi.org/10.11606/issn.2526-3358.bolzoo.1985.122293.

SAMPAIO, E.V., ROCHA, O., MATSUMURA-TUNDISI, T. and TUNDISI, J.G., 2002. Composition and abundance of zooplankton in the limnetic zone of seven reservoir of Paranapanema River, Brazil. Brazilian Journal of Biology $=$ Revista Brasileira de Biologia, vol. 62, no. 3, pp. 525-545. http://dx.doi.org/10.1590/ S1519-69842002000300018. PMid:12530191.

SARTORI, L.P., NOGUEIRA, M.G., HENRY, R. and MORETTO, E.M., 2009. Zooplankton fluctuations in Jurumirim Reservoir (São Paulo, Brazil): a three-year study. Brazilian Journal of Biology = Revista Brasileira de Biologia, vol. 69, no. 1, pp. 1-18. http:// dx.doi.org/10.1590/S1519-69842009000100002. PMid:19347141.

SENDACZ, S., CALEFFI, S. and SANTOS-SOARES, J., 2006. Zooplankton biomass of reservoirs in different trophic conditions in the State of São Paulo, Brazil. Brazilian Journal of Biology = Revista Brasileira de Biologia, vol. 66, no. 1B, pp. 337-350. http:// dx.doi.org/10.1590/S1519-69842006000200016. PMid:16710526.

SERAFIM-JÚNIOR, M., LANSAC-TÔHA, F.A., LOPES, R.M. and PERBICHE-NEVES, G., 2016. Continuity effects on rotifers and microcrustaceans caused by the construction of a downstream reservoir in a cascade series (Iguaçu River, Brazil). Brazilian Journal of Biology $=$ Revista Brasileira de Biologia, vol. 76, no. 2, pp. 279-291. http://dx.doi.org/10.1590/1519-6984.00314. PMid:26983084

SERAFIM-JÚNIOR, M., PERBICHE-NEVES, G., BRITO, L., GHIDINI, A.R. and CASANOVA, S.M.C., 2010. Variação espaço-temporal de Rotifera em um reservatório eutrofizado no sul do Brasil. Iheringia. Série Zoologia, vol. 100, no. 3, pp. 233-241. http://dx.doi.org/10.1590/S0073-47212010000300008.

SILVA, L.H.S., HUSZAR, V.L.M., MARINHO, M.M., RANGEL, L.M., BRASIL, J., DOMINGUES, C.D., BRANCO, C.C. and ROLAND, F., 2014. Drivers of phytoplankton, bacterioplankton, and zooplankton carbon biomass in tropical hydroelectric reservoirs. Limnologica, vol. 48, pp. 1-10. http://dx.doi.org/10.1016/j. limno.2014.04.004.

SIMÕES, N.R., NUNES, A.H., DIAS, J.D., LANSAC-TÔHA, F.A., VELHO, L.F.M. and BONECKER, C.C., 2015. Impact of reservoirs on zooplankton diversity and implications for the conservation of natural aquatic environments. Hydrobiologia, vol. 758, no. 1, pp. 3-17. http://dx.doi.org/10.1007/s10750-015-2260-y.

SOARES, M.C.S., MARINHO, M.M., HUSZAR, V.L.M., BRANCO, C.W. and AZEVEDO, M.F.O., 2008. The effects of water retention time and watershed features on the limnology 
of two tropical Reservoirs in Brazil. Lakes and Reservoirs: Research and Management, vol. 13, no. 4, pp. 257-269. http:// dx.doi.org/10.1111/j.1440-1770.2008.00379.x.

SOARES, M.C.S., VIDAL, L.O., ROLAND, F. and HUSZAR, V.L.M., 2009. Cyanobacterial equilibrium phases in a small tropical impoundment. Journal of Plankton Research, vol. 31, no. 11, pp. 1331-1338. http://dx.doi.org/10.1093/plankt/fbp079.

SOUSA, W., ATTAYDE, J.L., ROCHA, E.S. and ESKINAZISANT'ANNA, E.M., 2008. The response of zooplankton assemblages to variations in the water quality of four man-made lakes in semi-arid northeastern Brazil. Journal of Plankton Research, vol. 30, no. 6, pp. 699-708. http://dx.doi.org/10.1093/plankt/fbn032.

TAKAHASHI, E.M., LANSAC-TÔHA, F.A., DIAS, J.D., BONECKER, C.C. and VELHO, L.F.M., 2009. Spatial variations in the zooplankton community from the Corumbá Reservoir, Goiás State, in distinct hydrological periods. Acta Scientiarum. Biological Sciences, vol. 31, no. 3, pp. 227-234.

UTERMÖHL, H., 1958. Zur Vervollkommung der quantitativen Phytoplankton-Methodik. Mitteilungen der Internationalen Vereinigung für Theoretische und Angewandte Limnologie, vol. 9, pp. 1-38. 